PROCEEDINGS OF THE

AMERICAN MATHEMATICAL SOCIETY

Volume 127, Number 4, April 1999, Pages 1021-1028

S 0002-9939(99)04803-0

\title{
ORDINARY DIFFERENTIAL EQUATIONS WITH FRACTAL NOISE
}

\author{
F. KLINGENHÖFER AND M. ZÄHLE \\ (Communicated by Hal L. Smith) \\ To the memory of Johannes Kerstan
}

\begin{abstract}
The differential equation

$$
d x(t)=a(x(t), t) d Z(t)+b(x(t), t) d t
$$

for fractal-type functions $Z(t)$ is determined via fractional calculus. Under appropriate conditions we prove existence and uniqueness of a local solution by means of its representation $x(t)=h(y(t)+Z(t), t)$ for certain $C^{1}$-functions $h$ and $y$. The method is also applied to Itô stochastic differential equations and leads to a general pathwise representation. Finally we discuss fractal sample path properties of the solutions.
\end{abstract}

\section{INTRODUCTION}

In this paper we study the differential equation on the real line

$$
\begin{aligned}
d x(t) & =a(x(t), t) d Z(t)+b(x(t), t) d t, \\
x(0) & =x_{0}
\end{aligned}
$$

under the following conditions:

(C1) $Z$ is Hölder continuous of order $\lambda>\frac{1}{2}, Z(0)=0$,

(C2) $a \in C^{1}\left(\mathbb{R}^{2}, \mathbb{R}\right), a\left(x_{0}, 0\right) \neq 0$,

(C3) $b \in C\left(\mathbb{R}^{2}, \mathbb{R}\right)$ and $b$ is Lipschitz continuous in the first variable.

For vanishing $a$ this is a classical ordinary differential equation. Otherwise the term $d Z(t)$ adds a fractal noise which has to be made precise. The analogue in stochastic calculus is an Itô differential equation where $Z(t)$ is replaced by the Wiener process $W(t)$ or, more generally, by a semimartingale. In this language (1.1) may be interpreted as a differential equation driven by the fractal function $Z(t)$ with drift function $b$. Similarly as for the Itô case we give the differential equation (1.1) a strong mathematical sense via integration:

$$
x(t)=\int_{0}^{t} a(x(s), s) d Z(s)+\int_{0}^{t} b(x(s), s) d s .
$$

For the first fractal-type integral we use here the notion introduced in [4]. (Corresponding definitions and results will be summarized in section 2.) In particular, in [4] we proved calculation rules for the integral $\int_{a}^{b} f d g$ when $f$ and $g$ are Hölder

Received by the editors July 9, 1997.

1991 Mathematics Subject Classification. Primary 34A05; Secondary 60H10, 26A42.

(C)1999 American Mathematical Society 
continuous functions of summed order greater than 1 . It turns out that in this case the integral as a function of the boundary is Hölder continuous of the same order as $g$. Therefore it is appropriate to restrict the differential equation (1.1), i.e., the integral equation (1.2), to the space $H^{\lambda}$ of Hölder continuous functions of order $\lambda$ (given by that of $Z$ ). Within $H^{\lambda}$ we obtain the following main results:

(i) Existence of a local solution.

(ii) Uniqueness on the maximal interval of definition.

(iii) Representation of the solution in the form

$$
x(t)=h(y(t)+Z(t), t)
$$

where $h$ satisfies the quasilinear partial differential equation

$$
\begin{aligned}
h_{x}^{\prime}(x, t) & =a(h(x, t), t), \\
h(0,0) & =x_{0}
\end{aligned}
$$

and $y$ is the unique solution of the ordinary differential equation

$$
\begin{aligned}
& y^{\prime}(t)=\frac{b(h(Z(t)+y(t), t), t)-h_{t}^{\prime}(Z(t)+y(t), t)}{a(h(Z(t)+y(t), t), t)}, \\
& y(0)=0 .
\end{aligned}
$$

Furthermore, instead of deterministic $a, b$ and $Z$ we may choose arbitrary random functions with the same sample path properties and obtain local unique pathwise solutions. The practical relevance of this situation may be demonstrated on an example from finance mathematics. In order to take into consideration long range dependencies in real stock market processes the following model for the price $P$ of a risky asset appears to be appropriate:

$$
d P(t)=r(t) P(t) d t+\sigma(t) P(t) d B^{H}(t) .
$$

Here $r(t)$ and $\sigma(t)$ are called the interest rate and the dispersion function, respectively. $B^{H}$ denotes fractional Brownian motion with Hurst exponent $H>1 / 2$, i.e., a Gaussian process with stationary increments, mean zero and variance $\mathbb{E} B^{H}(t)^{2}=$ $t^{2 H}$. It possesses a version with Hölder continuous sample paths of all orders less than $H$. From our approach we obtain the unique global solution

$$
P(t)=P(0) \exp \left\{\int_{0}^{t} r(s) d s+\int_{0}^{t} \sigma(s) d B^{H}(s)\right\}
$$

(see also section 3). This problem has not been solved in the literature in full generality before.

In section 4 we will use the ideas from (1.3)-(1.5) in order to derive a similar representation for the solutions of one-dimensional Itô stochastic differential equations. The Wiener process $W$ as integrator has non-zero quadratic variation. Therefore the chain rule for the change of variables has to be replaced by the Itô formula which yields an additional term in (1.5).

At the end of this paper we discuss how the local representation of the solutions of the (stochastic) differential equations leads to information on the fractal structure of the sample paths. 


\section{Fractal integrals}

We first recall some definitions and notation from fractional calculus (cf. [3]).

For $f \in L_{1}([a, b])$ and $\alpha>0$ the left-sided and right-sided fractional RiemannLiouville integrals of $f$ of order $\alpha$ are defined at almost all $x$ by

$$
\begin{aligned}
I_{a+}^{\alpha} f(x) & =\frac{1}{\Gamma(\alpha)} \int_{a}^{x}(x-y)^{\alpha-1} f(y) d y \\
I_{b-}^{\alpha} f(x) & =\frac{(-1)^{-\alpha}}{\Gamma(\alpha)} \int_{x}^{b}(x-y)^{\alpha-1} f(y) d y .
\end{aligned}
$$

The class of functions $f$ which are representable as $I_{a+}^{\alpha}\left(I_{b-}^{\alpha}\right)$-integrals of some $L_{p^{-}}$function $\phi(p \geq 1)$ is denoted by $I_{a+}^{\alpha}\left(L_{p}\right)\left(I_{b-}^{\alpha}\left(L_{p}\right)\right.$, resp. $)$.

For $0<\alpha<1$ the function $\phi$ agrees at almost all $x$ with the Weyl-Marchaud derivative of $f$ order $\alpha$

$$
\begin{aligned}
D_{a+}^{\alpha} f(x) & =\frac{1}{\Gamma(1-\alpha)}\left(\frac{f(x)}{(x-a)^{\alpha}}+\alpha \int_{a}^{x} \frac{f(x)-f(y)}{(x-y)^{1+\alpha}} d y\right), \\
D_{b-}^{\alpha} f(x) & =\frac{(-1)^{\alpha}}{\Gamma(1-\alpha)}\left(\frac{f(x)}{(b-x)^{\alpha}}+\alpha \int_{x}^{b} \frac{f(x)-f(y)}{(y-x)^{1+\alpha}} d y\right) .
\end{aligned}
$$

Denote $f_{a+}=1_{(a, b)}(x)(f(x)-f(a+))$ and $f_{b-}=1_{(a, b)}(x)(f(x)-f(b-))$ assuming that the one-sided limits of $f$ at the interval ends exist.

Definition 2.1. The fractal integral of $f$ with respect to $g$ is defined by

$$
\begin{aligned}
\int_{a}^{b} f(x) d g(x)= & (-1)^{\alpha} \int_{a}^{b} D_{a+}^{\alpha} f_{a+}(x) D_{b-}^{1-\alpha} g_{b-}(x) d x \\
& +f(a+)(g(b-)-g(a+))
\end{aligned}
$$

provided that $f_{a+} \in I_{a+}^{\alpha}\left(L_{p}([a, b])\right), g_{b-} \in I_{b-}^{1-\alpha}\left(L_{q}([a, b])\right)$ for some $\frac{1}{p}+\frac{1}{q} \leq 1$ and $0 \leq \alpha \leq 1$.

This definition is shown to be independent of the choice of $\alpha$. It extends the classical Lebesgue-Stieltjes integral to functions of fractional degree of differentiability. Moreover, if $f \in H^{\mu}$ and $g \in H^{\nu}$ with $\mu+\nu>1$, then the uniform (but not absolute) convergence of the Riemann-Stieltjes sums to the right hand side of (2.5) is proved. Thus, in this case our integral may be interpreted as a Riemann-Stieltjes integral. (In the proof we have essentially used the representation by fractional derivatives.) This interpretation leads to the chain rule for the change of variables:

Let $F \in C^{1}\left(\mathbb{R}^{2}, \mathbb{R}\right)$ be such that $\frac{\partial F}{\partial x_{1}}(f(\cdot), \cdot)$ is Hölder continuous of order $\mu$. Then we have

$$
F(f(y), y)-F(f(a), a)=\int_{a}^{y} \frac{\partial F}{\partial x_{1}}(f(x), x) d f(x)+\int_{a}^{y} \frac{\partial F}{\partial x_{2}}(f(x), x) d x .
$$

Furthermore, the integral $\phi=\int_{a}^{(\cdot)} f d g$ as a function of the upper boundary is Hölder continuous of order $\nu$.

Taking $\phi$ as a new integrator we obtain the density-type formula

$$
\int_{a}^{b} h d \phi=\int_{a}^{b} f h d g
$$

for any $h \in H^{\mu}$.

For the proofs, more details and extensions see [4]. 


\section{Solution of the Differential EQUATION}

We now return to equation (1.2) under the conditions $(\mathrm{C} 1)-(\mathrm{C} 3)$. In order to prove local existence and uniqueness of the solution we will show that any function of the form (1.3) provides a solution of (1.2) and that all solutions locally agree with such a fixed function.

Proposition 3.1. There exist some constants $t_{1}>0, K>0$ and a function $h \in$ $C^{1}\left((-K, K) \times\left(-t_{1}, t_{1}\right), \mathbb{R}\right)$ solving the quasilinear partial differential equation (1.4):

$$
\begin{aligned}
h_{x}^{\prime}(x, t) & =a(h(x, t), t), \\
h(0,0) & =x_{0} .
\end{aligned}
$$

For any such $h$ the partial derivatives $h_{x x}^{\prime \prime}$ and $h_{x t}^{\prime \prime}=\frac{\partial}{\partial x} h_{t}^{\prime}$ exist and yield continuous functions.

Proof. The first part is standard in the theory of first-order partial differential equations and we omit the proof. The existence and continuity of $h_{x x}^{\prime \prime}$ is obvious. Further, by means of the representation

$$
h(x, t)=h(0, t)+\int_{0}^{x} a(h(y, t), t) d y
$$

we get

$$
h_{t}^{\prime}(x, t)=h_{t}^{\prime}(0, t)+\int_{0}^{x}\left(a_{x}^{\prime}(h(y, t), t) h_{t}^{\prime}(y, t)+a_{t}^{\prime}(h(y, t), t)\right) d y
$$

since the partial derivatives under the integral are continuous. Hence,

$$
h_{x t}^{\prime \prime}(x, t)=a_{x}^{\prime}(h(x, t), t) h_{t}^{\prime}(x, t)+a_{t}^{\prime}(h(x, t), t) .
$$

Let us now fix such a solution $h$ with $t_{1}$ and $K$ as above.

Proposition 3.2. There exist some $0<t_{2} \leq t_{1}$ and a function $y \in C^{1}\left(\left(-t_{2}, t_{2}\right), \mathbb{R}\right)$ with $|y(t)+Z(t)|<K, t \in\left(-t_{2}, t_{2}\right)$, satisfying the $O D E$ (1.5), i.e.,

$$
\begin{aligned}
& y^{\prime}(t)=\frac{b(h(Z(t)+y(t), t), t)-h_{t}(Z(t)+y(t), t)}{a(h(Z(t)+y(t), t), t)}, \\
& y(0)=0 .
\end{aligned}
$$

Any two such solutions agree on all common intervals of definition.

The proof is standard in the theory of ODE using the conditions (C1)-(C3) together with the $C^{1}$-property of $h_{t}^{\prime}$ with respect to the first argument and applying Banach's fixed point theorem.

Our main result may now be formulated as follows.

Theorem 3.3. (i) Any function

$$
x(t)=h(y(t)+Z(t), t)
$$

with $h$ and $y$ as in (3.1) and (3.2) solves the differential equation (1.1).

(ii) Any two solutions of (1.1) agree on all common intervals of definition as long as $a(x(t), t) \neq 0$. 
Proof. (i) follows from the change-of-variable formula (2.6) applied to the function $F(x, t):=h(y(t)+x, t)$ for $t \in\left(-t_{2}, t_{2}\right)$ :

$$
\begin{aligned}
h(y(t) & +Z(t), t)-h(0,0) \\
= & \int_{0}^{t} h_{x}^{\prime}(y(s)+Z(s), s) d Z(s) \\
& \quad+\int_{0}^{t}\left(h_{x}^{\prime}(y(s)+Z(s), s) y^{\prime}(s)+h_{t}^{\prime}(y(s)+Z(s), s)\right) d s \\
= & \int_{0}^{t} a(h(y(s)+Z(s), s), s) d Z(s)+\int_{0}^{t} b(h(y(s)+Z(s), s), s) d s .
\end{aligned}
$$

In order to prove (ii) let $x(t)=h(y(t)+Z(t), t)$ be as above and $\bar{x}(t)$ be an arbitrary solution of (1.1). By the inverse function theorem and condition (C2) there exist some $t_{3} \leq t_{2}$ and a $C^{1}\left((-K, K) \times\left(-t_{3}, t_{3}\right),(-K, K)\right)$-function $u$ such that $(x, t) \rightarrow$ $(u(x, t), t)$ is the inverse of the mapping $(x, t) \rightarrow(h(x, t), t)$. In particular, $u\left(x_{0}, 0\right)=$ 0 and the partial derivatives of $u$ are given by

$$
\begin{aligned}
u_{x}^{\prime}(x, t) & =h_{x}^{\prime}(u(x, t), t)^{-1}=a(x, t)^{-1} \\
u_{t}^{\prime}(x, t) & =-h_{t}^{\prime}(u(x, t), t) h_{x}^{\prime}(u(x, t), t)^{-1} \\
& =-h_{t}^{\prime}(u(x, t), t) a(x, t)^{-1} .
\end{aligned}
$$

Note that $u_{x}^{\prime}$ has a continuous partial derivative $u_{x x}^{\prime \prime}$ as long as $a(u(x, t), t) \neq 0$. Therefore we may apply the change-of-variable formula to $F(x, t):=u(x, t)+y(t)$ for all sufficiently small $t$ regarding that $u(\bar{x}(0), 0)+y(0)=u\left(x_{0}, 0\right)=0$ :

$$
\begin{aligned}
u(\bar{x}(t), t) & =\int_{0}^{t} u_{x}^{\prime}(\bar{x}(s), s) d \bar{x}(s)+\int_{0}^{t} u_{t}^{\prime}(\bar{x}(s), s) d s \\
& =\int_{0}^{t} a(\bar{x}(s), s)^{-1} d \bar{x}(s)-\int_{0}^{t} \frac{h_{t}^{\prime}(u(\bar{x}(s), s), s)}{a(\bar{x}(s), s)} d s .
\end{aligned}
$$

Using $d \bar{x}(t)=a(\bar{x}(t), t) d Z(t)+b(\bar{x}(t), t) d t$ and the density-type formula (2.6) we obtain from this

$$
u(\bar{x}(t), t)=Z(t)+\int_{0}^{t} \frac{b(\bar{x}(s), s)-h_{t}^{\prime}(u(\bar{x}(s), s), s)}{a(\bar{x}(s), s)} d s .
$$

Denoting $\bar{y}(t):=u(\bar{x}(t), t)-Z(t)$ we infer

$$
\bar{y}(t)=\int_{0}^{t} \frac{b(h(\bar{y}(s)+Z(s), s), s)-h_{t}^{\prime}(\bar{y}(s), s)}{a(h(\bar{y}(s)+Z(s), s), s)} d s,
$$

i.e., $\bar{y}$ is a local solution of (1.5) as in Proposition 3.2. Therefore it agrees locally with $y(t)$. Hence,

$$
\begin{aligned}
\bar{x}(t) & =h(u(\bar{x}(t), t), t)=h(\bar{y}(t)+Z(t), t) \\
& =h(y(t)+Z(t), t)=x(t)
\end{aligned}
$$

for all sufficiently small $t$.

Since we may replace the moment $t=0$ by an arbitrary $t_{0}$ with $a\left(x\left(t_{0}\right), t_{0}\right) \neq 0$ (and $x_{0}$ by $\left.x\left(t_{0}\right)\right)$ the assertion follows.

Remark 3.4. In the time autonomous case $a(x, t)=a(x), b(x, t)=b(x)$ the local solution of (1.1) takes the form

$$
x(t)=h(y(t)+Z(t))
$$


with $C^{1}$-functions $h$ and $y$ determined by the ODE

$$
\begin{aligned}
h^{\prime}(x) & =a(h(x)), \\
h(0) & =x_{0}
\end{aligned}
$$

and

$$
\begin{aligned}
y^{\prime}(t) & =\frac{b(h(y(t)+Z(t)))}{a(h(y(t)+Z(t)))}, \\
y(0) & =0
\end{aligned}
$$

respectively.

In particular, we can take for $Z(t)$ the sample paths of an ergodic stationary random process which are assumed to be $\lambda$-Hölder continuous with probability 1. If $b \equiv 0$, then the resulting random process $X(t)=h(Z(t))$ is stationary and ergodic, too. This answers a question posed by M. Keane.

\section{Solution of Itô Stochastic Differential equations}

As a by-product of the techniques developed in this paper we obtain a new local representation of the solution of one-dimensional stochastic differential equations in the classical Itô sense. We suppose that the reader is familiar with the corresponding theory (see, e.g., Karatzas and Shreve [1] for related material). The SDE under consideration is of the form

$$
X(t)=X_{0}+\int_{0}^{t} a(X(s), s) d W(s)+\int_{0}^{t} b(X(s), s) d s,
$$

where $W(t)$ denotes the standard Wiener process and the first integral on the right hand side is defined in the Itô sense. Here we suppose the following:

$\left(\mathrm{C} 1^{\prime}\right) X_{0}$ is a random variable independent of $\left\{W_{t}\right\}_{t>0}$.

$\left(\mathrm{C} 2^{\prime}\right) a \in C^{1}\left(\mathbb{R}^{2}, \mathbb{R}\right), a_{x}^{\prime}$ is Lipschitz continuous in the first variable and $a\left(X_{0}, 0\right) \neq 0$ with probability 1 .

$\left(\mathrm{C} 3^{\prime}\right) b \in C\left(\mathbb{R}^{2}, \mathbb{R}\right)$ and $b$ is Lipschitz continuous in the first variable.

Similarly as in Proposition 3.1 we obtain for a fixed value of $X_{0}$ and for some constants $K>0, t_{1}>0$ a function $h \in C^{1}\left((-K, K) \times\left[0, t_{1}\right), \mathbb{R}\right)$ solving the quasilinear partial differential equation

$$
\begin{aligned}
h_{x}^{\prime}(x, t) & =a(h(x, t), t), \\
h(0,0) & =X_{0} .
\end{aligned}
$$

The partial derivatives $h_{x t}^{\prime \prime}$ and $h_{x x}^{\prime \prime}$ are continuous functions and the latter is Lipschitz continuous in the first variable.

The analogue of Proposition 3.2 reads as follows: There exist a random stopping time $\tau \in\left(0, t_{1}\right)$ and a unique adapted process $Y$ with respect to the filtration given by $X_{0}$ and $W(t)$ such that $Y$ is continuously differentiable and

$$
\begin{aligned}
Y^{\prime}(t) & =\frac{b(h(W(t)+Y(t), t), t)-h_{t}^{\prime}(W(t)+Y(t), t)-\frac{1}{2} h_{x x}^{\prime \prime}(W(t)+Y(t), t)}{a(h(W(t)+Y(t), t), t)}, \\
Y(0) & =0
\end{aligned}
$$

on the interval $[0, \tau)$ with probability 1 . 
Now let $h, \tau$ and $Y$ be given.

Theorem 4.1. The stochastic process

$$
X(t)=h(Y(t)+W(t), t), t \in[0, \tau),
$$

is a solution of (4.1).

Remark 4.2. Under the conditions $\left(\mathrm{C}^{\prime}\right)-\left(\mathrm{C} 3^{\prime}\right)$ existence and uniqueness of a strong solution is well-known (cf. [1]). If we assume $a \in C^{2}\left(\mathbb{R}^{2}, \mathbb{R}_{+}\right)$, the local uniqueness may also be proved by the methods of section 3 .

Proof of Theorem 4.1. The Itô formula implies:

$$
\begin{aligned}
h(Y(t) & +W(t), t)-h(Y(0), 0) \\
= & \int_{0}^{\tau \wedge t} h_{x}^{\prime}(Y(s)+W(s), s) d W(s) \\
& +\int_{0}^{\tau \wedge t}\left(h_{x}^{\prime}(Y(s)+W(s), s) Y^{\prime}(s)+h_{t}^{\prime}(Y(s)+W(s), s)\right) d s \\
& +\frac{1}{2} \int_{0}^{\tau \wedge t} h_{x x}^{\prime \prime}(Y(s)+W(s), s) d s \\
= & \int_{0}^{\tau \wedge t} a(h(Y(s)+W(s), s), s) d W(s)+\int_{0}^{\tau \wedge t} b(h(Y(s)+W(s), s), s) d s .
\end{aligned}
$$

In a subsequent paper this pathwise representation of the solution will be extended to the higher-dimensional anticipating case with nilpotent Lie algebra generated by the vector fields, where the stochastic integrals are introduced in an appropriate way. There the function $h$ depends also on the iterated integrals of Brownian motion.

\section{FRACTAL SAMPLE PATH PROPERTIES}

The local representations

$$
x(t)=h(y(t)+Z(t), t)
$$

and

$$
X(t)=h(Y(t)+W(t), t)
$$

of the solution of (1.1) and (4.1), respectively, provide full information on some fractal sample path properties:

Recall that the functions $h, y$ and $Y$ are continuously differentiable and $h$ is locally $C^{1}$-invertible in the first variable (cf. the proof of Theorem 3.3 ). Therefore we obtain a local bi-Lipschitz connection between the graph of the input function $Z(t)$ (or $W(t)$ ) and the output function $x(t)$ (or $X(t)$ ) of the dynamical system. This implies that fractal characteristics such as Hausdorff and packing dimensions of the graph, local Hölder exponent, fractional degree of differentiability, etc., of the input and output functions are the same. In particular, the diffusion processes $X(t)$ under the above conditions possess the same well-known properties as the Wiener process. 
Note added in proof. Equation (1.1) is also treated in [2] in the context of RiemannStieltjes integrals for functions of finite $p$-variation $(p<2)$ via Picard's iteration procedure.

\section{REFERENCES}

1. I. Karatzas and E. Shreve, Brownian motion and stochastic calculus. Springer, New York, 1991. MR 92h:60127

2. T.G. Lyons, Differential equations driven by rough signals (I): an extension of an inequality by L.C. Young, Mathematical Research Letters 1 (1994), 451-464. MR 96b:60150

3. S.G. Samko, A.A. Kilbas, and O. Marichev, Fractional Integrals and Derivatives. Theory and Applications. Gordon and Breach, New York, 1993. MR 96d:26012

4. M. Zähle, Integration with Respect to Fractal Functions and Stochastic Calculus, Probab. Theory Related Fields (to appear).

Mathematical Institute, University of Jena, D-07740 Jena, Germany

E-mail address: klingenhofer@minet.uni-jena.de

E-mail address: zaehle@minet.uni-jena.de 